\title{
LAKE ICE DETECTION IN LOW-RESOLUTION OPTICAL SATELLITE IMAGES
}

\author{
Manu Tom ${ }^{1 *}$, Ursula Kälin ${ }^{1}$, Melanie Sütterlin², Emmanuel Baltsavias ${ }^{1}$, Konrad Schindler $^{1}$ \\ ${ }^{1}$ Photogrammetry and Remote Sensing Group, ETH Zürich, Switzerland \\ (manu.tom, manos, schindler)@geod.baug.ethz.ch, kaelinu@student.ethz.ch \\ ${ }^{2}$ Institute of Geography, University of Bern, Switzerland \\ melanie.suetterlin@giub.unibe.ch
}

Commission II, ICWG II/III

KEY WORDS: VIIRS, MODIS, SVM, Lake Ice, Semantic Segmentation, Climate Change

\begin{abstract}
:
Monitoring and analyzing the (decreasing) trends in lake freezing provides important information for climate research. Multi-temporal satellite images are a natural data source to survey ice on lakes. In this paper, we describe a method for lake ice monitoring, which uses low spatial resolution $(250 \mathrm{~m}-1000 \mathrm{~m})$ satellite images to determine whether a lake is frozen or not. We report results on four selected lakes in Switzerland: Sihl, Sils, Silvaplana and St. Moritz. These lakes have different properties regarding area, altitude, surrounding topography and freezing frequency, describing cases of medium to high difficulty. Digitized Open Street Map (OSM) lake outlines are back-projected on to the image space after generalization. As a pre-processing step, the absolute geolocation error of the lake outlines is corrected by matching the projected outlines to the images. We define the lake ice detection as a two-class (frozen, non-frozen) semantic segmentation problem. Several spectral channels of the multi-spectral satellite data are used, both reflective and emissive (thermal). Only the cloud-free (clean) pixels which lie completely inside the lake are analyzed. The most useful channels to solve the problem are selected with $x$ gboost and visual analysis of histograms of reference data, while the classification is done with non-linear support vector machine (SVM). We show experimentally that this straight-forward approach works well with both MODIS and VIIRS satellite imagery. Moreover, we show that the algorithm produces consistent results when tested on data from multiple winters.
\end{abstract}

\section{INTRODUCTION}

The Global Climate Observing System (GCOS) is one of the programmes initiated by the World Meteorological Organization with a vision to provide all users access to climate observations, data records and information. Lake Ice Cover is part of the GCOS Essential Climate Variable (ECV): Lakes. Analyzing lake ice trends is significant for climate change research and global warming studies. In addition, surveillance of the lake ice fosters development of winter tourism (e.g., for snow polo world cup on frozen St. Moritz lake). Traditionally, on-shore observers used to collect the information on frozen lakes, recording the visible ice-edge. Over the past few decades the number of field stations declined, due to lack of budget and/or human resources. Day by day, the need for automated monitoring of lakes is gaining significance. There exist already observations and data from regional authorities, publications etc., however they are not systematic and come from different, uncoordinated and not secure sources. In this paper, we report results for four Alpine lakes shown in Figure 1. These lakes have different attributes regarding area $(0.78$ to $11.3 \mathrm{~km}^{2}$ ), neighboring topography, freezing and thawing patterns and altitude.

Contributions. This paper describes an application study in the context of the Swiss input to GCOS. Its key contributions can be summarized as follows: (1) We demonstrate an easy-to-use, SVM-based approach for lake ice detection. (2) We estimate and compare the absolute geolocation error for both MODIS and VIIRS sensors. (3) We show that the proposed approach delivers convincing results with both MODIS and VIIRS satellite images, for various lake types and time periods.

\footnotetext{
${ }^{*}$ Corresponding author.
}

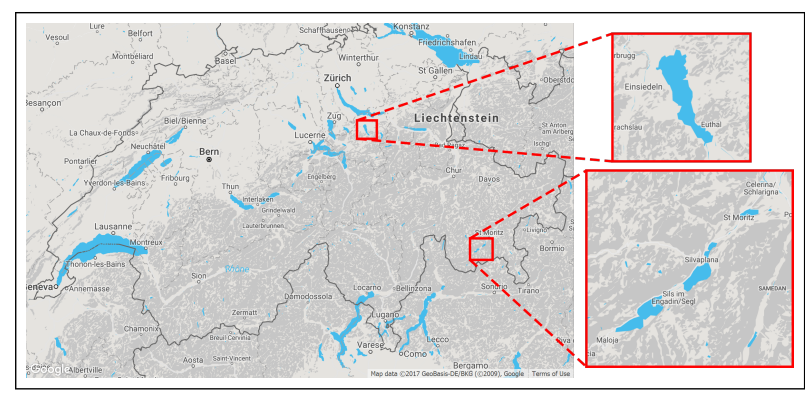

Figure 1: Left image: map of Switzerland with target lakes displayed in blue. The top-right image shows lake Sihl, the bottom-right image the region of lakes Sils, Silvaplana and St. Moritz (from left to right). Image courtesy of Google. Best viewed on screen.

\section{RELATED WORK}

Monitoring of lakes. Studies on lake ice exist for several countries, including Spain (Sánchez-López et al., 2015), Germany (Bernhardt et al., 2012), Austria and Hungary (Soja et al., 2014), and Tibet (Korzeniowska and Korup, 2017). An investigation of the recent developments in lake remote sensing (Dörnhöfer and Oppelt, 2016) discusses existing challenges and benefits of integrating remote sensing into ecological research and lake monitoring. Various studies also exist in the literature which delve deep into the monitoring of lake ice using different sensors (Maslanik and Barry, 1987; Palecki and Barry, 1986; Wynne and Lillesand, 1993). A recent paper (Weber et al., 2016) discusses an automated two-step extraction method for ice phenology in European lakes using AVHRR data. The first step uses NIR reflectance and the second step uses TIR-derived Lake Surface Water Temperature (LSWT) data. The LSWT thresholds are derived from the data itself which avoids the usage of lake specific thresholds. 
ISPRS Annals of the Photogrammetry, Remote Sensing and Spatial Information Sciences, Volume IV-2, 2018 ISPRS TC II Mid-term Symposium “Towards Photogrammetry 2020”, 4-7 June 2018, Riva del Garda, Italy

\begin{tabular}{|c|c|c|c|c|c|c|c|c|c|c|c|c|}
\hline Band & $B_{1}$ & $B_{2}$ & $B_{3}$ & $B_{4}$ & $B_{6}$ & $B_{17}$ & $B_{18}$ & $B_{19}$ & $B_{20}$ & $B_{22}$ & $B_{23}$ & $B_{25}$ \\
\hline central wavelength $(\mu m)$ & 0.645 & 0.858 & 0.469 & 0.555 & 1.64 & 0.905 & 0.936 & 0.940 & 3.75 & 3.96 & 4.05 & 4.52 \\
bandwidth $(\mu m)$ & 0.05 & 0.04 & 0.02 & 0.02 & 0.02 & 0.03 & 0.01 & 0.05 & 0.18 & 0.06 & 0.06 & 0.07 \\
type & $\mathrm{R}$ & $\mathrm{R}$ & $\mathrm{R}$ & $\mathrm{R}$ & $\mathrm{R}$ & $\mathrm{R}$ & $\mathrm{R}$ & $\mathrm{R}$ & $\mathrm{E}$ & $\mathrm{E}$ & $\mathrm{E}$ & $\mathrm{E}$ \\
GSD (m) & 250 & 250 & 500 & 500 & 500 & 1000 & 1000 & 1000 & 1000 & 1000 & 1000 & 1000 \\
\hline
\end{tabular}

Table 1: The 12 potentially useful MODIS reflective (R) and emissive (E) channels, with their respective central wavelength, bandwidth and pixel Ground Sampling Distance (GSD).

MODIS for lake ice. In south-west Alaska, the inter-annual variability in snow pack, lake ice, and vegetation dynamics were assessed using MODIS images (Spencer et al., 2008). According to this study, lake ice showed the greatest temporal variation among the observed seasonal metrics, followed by snow cover and vegetation metrics. MODIS images are also useful for estimating melt pond coverage on the Arctic sea ice (Tschudi et al., 2008). In that work, a mixed-pixel algorithm to estimate the summer evolution of pond coverage over the Beaufort and Chukchi Seas has been proposed. In another work (Rösel et al., 2012), melt ponds on the Arctic sea ice were determined in MODIS images by spectral unmixing using an artificial neural network. There exists yet another study which showed that the MODIS product is useful for monitoring daily ice cover changes in lakes, and to obtain ice-on and ice-off dates (Brown and Duguay, 2012). For the period of 2000-2014, the data from Terra MODIS was utilized to determine variations in Minimum Snow and Ice (MSI) extent over the land surfaces covering Canada, Northern United States, and Iceland (Trishchenko et al., 2016). Using MODIS imagery, an automated ice detection methodology revealed the spatial and temporal patterns in the Arctic river ice breakup (Cooley and Pavelsky, 2016). In the recent past, a probabilistic approach for mapping landfast and sea ice extent in the Canadian Arctic Archipelago from MODIS images was also proposed ( $\mathrm{Tr}-$ ishchenko et al., 2017).

VIIRS-based approaches. As opposed to MODIS imagery, the VIIRS data has not been explored much for lake ice analysis. Recently, the retrievals of LSWT and ice features from corrected clear-sky channel $\left(I_{5}\right)$ data of the VIIRS sensor were proposed (Sütterlin et al., 2017). Together with the visible and near infrared reflectance values, these first LSWT retrievals were used to derive ice-on/off dates for selected Swiss lakes by applying a threshold method. Another algorithm based on VIIRS images (Liu et al., 2016) detected ice and estimated the ice concentration in clear-sky areas over the ocean and inland lakes and rivers, providing spatial detail that cannot be obtained with passive microwave data. An inter-comparison of VIIRS and MODIS (Trishchenko and Ungureanu, 2017) has also been carried out. This comparison is done primarily based on the mapping results of summer MSI extent over the Canadian landmass.

\section{DATA}

The used satellite images are freely available and can be processed fast, with a processing chain using standard hardware and software tools. Both MODIS and VIIRS have high temporal resolution (at least one image per day), sufficient spectral resolution, but rather coarse spatial resolution (GSD 250m-1000m). Here, we process only daytime acquisitions, for both VIIRS and MODIS.

\subsection{MODIS}

Two copies of MODIS are in orbit onboard the Terra (Terra Mission, EOS/AM-1) and Aqua satellites. In this work, we use only Terra MODIS imagery, as Aqua MODIS images have inferior quality and shorter time archives. The MODIS sensor captures data in 36 spectral channels and at varying spatial resolutions (2 bands with $250 \mathrm{~m}$ at nadir, 5 bands with $500 \mathrm{~m}$ at nadir and 29 bands with $1000 \mathrm{~m}$ at nadir). We used the following MODIS products: MOD02 (level 1B, calibrated and geolocated radiances), MOD03 (geolocation) and MOD35 (48-bit cloud-mask). The MODIS data was downloaded from the LAADS DAAC (Level-1 and Atmosphere Archive \& Distribution System Distributed Active Archive Center) archive. After a visual check, 12 bands (Table 1 ) were selected as potentially useful. The remaining 24 channels were discarded either due to saturation issues, or presence of stripes. MODIS data was processed from the following two winters: $2011-12$ and 2016-17. The respective details about the data are described in the sections 3.3.1 and 3.3.2. The details of MODIS ground truth data for the periods 2016-17 and 2011-12 are displayed in Table 2 and Table 3 respectively.

\subsection{VIIRS}

The VIIRS aboard the joint NASA/NOAA Suomi NPP satellite (Suomi National Polar-orbiting Partnership Mission, n.d.), gathers visible and infrared imagery and radiometric measurements of the land, atmosphere, cryosphere, and oceans. The VIIRS sensor captures 16 moderate-resolution bands (M-bands) with a spatial resolution of $750 \mathrm{~m}$ at nadir, and five imaging resolution bands (I-bands or imagery bands) with $\sim 375 \mathrm{~m}$ spatial resolution at nadir. However, the scope of this work is limited to the analysis of the I-bands to allow a better comparison with another method of lake ice detection from VIIRS developed by the University of Bern, using only the I-bands. These imagery bands include three Reflective Solar Bands (RSB) and two Thermal Emissive Bands (TEB). Details are shown on Table 4. VIIRS data is downloaded from the Comprehensive Large Array Stewardship System (CLASS) archive of NOAA. We used the VIIRS imaging resolution bands Scientific Data Record (SDR), cloud-masks (IICMO and VICMO) and geolocation (GITCO). VIIRS data was processed only from the winter $2016-17$, for which the ground truth data is displayed on Table 2 and discussed in section 3.3.1.

\subsection{Ground Truth}

We mainly used freely available images from webcams pointing at target lakes to obtain the ground truth. Given the fact that the webcams do not cover the complete lake surface, and their scale (pixel GSD) varies greatly for each webcam, manual interpretation is in some cases uncertain. At present, we have restricted our analysis to the days on which the lakes were completely frozen or completely non-frozen. In addition to the webcam images we used online media reports, which however provided only scarce information.

3.3.1 Winter 2016-17. For lakes Sihl, Sils, and Silvaplana, the non-frozen data (for both MODIS and VIIRS) comprises of the acquisitions from October 2016 and first half of December 2016. The second half of December 2016 was not analyzed due to the uncertain interpretation of the ground truth (freezing period with water and thin ice). For lake St. Moritz, in December 2016, only the acquisitions until 09th of December were included. This is because St. Moritz started to freeze (partially) from 10th of December. St. Moritz was processed only using MODIS. For all the lakes, the frozen data (both MODIS and VIIRS) contains the acquisitions from February 2017. From the webcam images, we 
ISPRS Annals of the Photogrammetry, Remote Sensing and Spatial Information Sciences, Volume IV-2, 2018 ISPRS TC II Mid-term Symposium “Towards Photogrammetry 2020”, 4-7 June 2018, Riva del Garda, Italy

\begin{tabular}{|c|c|c|c|c|c|c|c|c|c|c|c|c|c|c|c|c|}
\hline & \multicolumn{4}{|c|}{ Lake Sihl } & \multicolumn{4}{|c|}{ Lake Sils } & \multicolumn{4}{|c|}{ Lake Silvaplana } & \multicolumn{4}{|c|}{ Lake St. Moritz } \\
\hline & \multicolumn{2}{|c|}{ MODIS } & \multicolumn{2}{|c|}{ VIIRS } & \multicolumn{2}{|c|}{ MODIS } & \multicolumn{2}{|c|}{ VIIRS } & \multicolumn{2}{|c|}{ MODIS } & \multicolumn{2}{|c|}{ VIIRS } & \multicolumn{2}{|c|}{ MODIS } & \multicolumn{2}{|c|}{ VIIRS } \\
\hline & $\mathbf{A q}$ & Px & Aq & $\mathbf{P x}$ & Aq & Px & $\mathbf{A q}$ & Px & $\mathbf{A q}$ & Px & Aq & Px & $\mathbf{A q}$ & Px & $\mathbf{A q}$ & $\mathbf{P x}$ \\
\hline Frozen & 7 & 805 & 19 & 774 & 8 & 263 & 15 & 153 & 11 & 227 & 17 & 140 & 9 & 36 & - & - \\
\hline Non-Frozen & 17 & 1809 & 29 & 1222 & 15 & 471 & 32 & 328 & 18 & 349 & 32 & 289 & 13 & 52 & - & - \\
\hline
\end{tabular}

Table 2: Available ground truth data on cloud-free dates processed in winter 2016-17. Frozen data comprises of the acquisitions from February 2017, non-frozen data from October 2016 and December 2016. Only the first half of December 2016 are processed. The table shows the total number of clean, cloud-free pixels ( $\mathrm{Px})$ and the total number of acquisitions (Aq) which are $>30 \%$ cloud-free.

\begin{tabular}{|c|c|c|c|c|c|c|c|c|c|c|c|c|}
\hline & \multicolumn{3}{|c|}{ Lake Sihl } & \multicolumn{3}{c|}{ Lake Sils } & \multicolumn{3}{c|}{ Lake Silvaplana } & \multicolumn{3}{c|}{ Lake St. Moritz } \\
\cline { 2 - 13 } & Period & Aq & Px & Period & Aq & Px & Period & Aq & Px & Period & Aq & Px \\
\hline Frozen & $06-25$ Feb.12 & 7 & 662 & Jan, Feb.12 & 23 & 656 & Jan, Feb.12 & 23 & 479 & Jan, Feb.12 & 23 & 92 \\
Non-Frozen & $20-31$ Mar.12 & 6 & 648 & Nov.11 & 15 & 443 & Nov.11 & 15 & 300 & Nov.11 & 14 & 56 \\
\hline
\end{tabular}

Table 3: MODIS ground truth information on the cloud-free dates processed in 2011-12.

\begin{tabular}{|c|c|c|c|}
\hline Band & Spectrum & Cent. wavel. $(\mu m)$ & BW $(\mu m)$ \\
\hline$I_{1}$ & Visible Near IR & 0.640 & 0.05 \\
$I_{2}$ & Visible Near IR & 0.865 & 0.04 \\
$I_{3}$ & Short-Wave IR & 1.61 & 0.06 \\
$I_{4}$ & Mid-Wave IR & 3.74 & 0.38 \\
$I_{5}$ & Thermal IR & 11.450 & 1.90 \\
\hline
\end{tabular}

Table 4: VIIRS I-channels, the corresponding spectral regions, center wavelength and bandwidth (BW).

\begin{tabular}{|c|c|c|c|}
\hline & $\mu_{x}$ (pixel) & $\mu_{y}$ (pixel) & Study period \\
\hline MODIS & 0.75 & 0.85 & Dec.2011 - Mar.2012 \\
VIIRS & 0.0 & 0.3 & Oct.2016, Dec.2016 \\
\hline
\end{tabular}

Table 5: Mean shifts in $\mathrm{x}\left(\mu_{x}\right)$ and $\mathrm{y}\left(\mu_{y}\right)$ directions (estimated absolute geolocation errors) for both sensors.

confirmed that all lakes were frozen with snow on top of the ice. An exception is lake Sihl which had ice until 05th of February and snow on ice afterwards.

3.3.2 Winter 2011-12. We could not obtain webcam data for the winter 2011-12. Hence, the scarce ground truth is obtained primarily from the online media. The three lakes in Graubünden typically start to freeze in December and thaw around end of March. In addition, given the fact that winter 2011-12 was the coldest among the recent winters, we made the assumption that the three smaller lakes in Canton Graubünden (Sils, Silvaplana and St. Moritz) were frozen in the months January 2012 and February 2012, based on information that larger lakes at lower altitude were also frozen in January and February. For lake Sihl, we gathered adequate information on the ground truth from the online media. Lake Sihl was covered by snow on all frozen dates which we tested in winter 2011-12 (Feb 06, 2012 - Feb 25, 2012). Hence, for lake Sihl, effectively it is snow vs. water classification. However, the other three lakes had ice without snow on some days in January 2012 (only few days for lake St. Moritz) which makes the 2011-12 dataset more challenging than 2016-17.

\section{METHODOLOGY}

\subsection{Pre-processing}

The VIIRS pre-processing includes python modules from Pytroll (VIIRS with Pytroll, n.d.), which is a free and open source software suited for most of the pre-processing steps (i.e., reading, resampling, interpretation, and writing) of satellite data. The raw VIIRS data granules were assembled, mapped, and re-sampled. Samples with fill-in values resulting from bow-tie deletion were removed. Similarly, the MODIS raw data was re-sampled and re-projected to UTM $32 \mathrm{~N}$ coordinates.

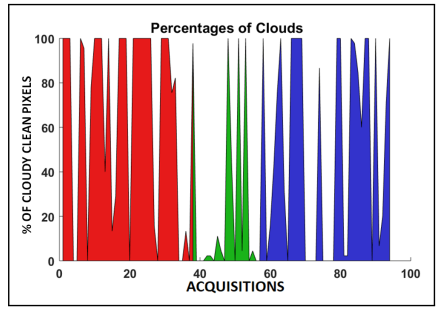

Figure 2: VIIRS cloud-cover for lake Sihl. Acquisitions in October 2016 are shown in red, first half of December 2016 in green and February 2017 in blue. Best viewed on screen.

Absolute Geolocation Correction. The digitized OSM lake outlines were generalized using Douglas-Peucker method and corrected for absolute geolocation errors prior to back-projection on the image space. The absolute geolocation shifts for both VIIRS and MODIS data were estimated using the lake matching algorithm (Kocaman Aksakal, 2013). Channels with the best contrast ( $I_{2}$ for VIIRS and $B_{2}$ for MODIS) were fed as input to the algorithm. For this analysis, we used the 15 largest lakes in and around Switzerland. Data from two nearly cloud-free dates per month were used. MODIS shifts were estimated from four months in winter 2011-12. On the other hand, VIIRS shifts were estimated from two months in winter 2016-17. Table 5 displays the estimated final shifts for VIIRS and MODIS. It can be seen that the absolute geolocation of the VIIRS images is more accurate than the one of MODIS. The specified geolocation accuracy of MODIS is $300 \mathrm{~m}(\approx 1.2$ pixels) at nadir (Nishihama et al., 1997).

Cloud Filtering. An inherent challenge of optical satellite image analysis is missing data due to cloud cover. Figure 2 illustrates the extent of this issue. For each acquisition on the $\mathrm{x}$-axis, the corresponding percentage of clean cloudy pixels is shown on the y-axis, per lake. It can be seen that a lot of acquisitions are unusable due to the presence of clouds. Images with more than $70 \%$ cloudy pixels are not analyzed. Each MODIS channel has 16-bit grey values of which the invalid pixels have value above 32767 . For all other images, cloudy and invalid pixels are masked out prior to analysis. Cloud detection performance can vary from one sensor to another. Separate cloud-masks are produced for MODIS and VIIRS. Snow/cloud confusion and large view angles can result in considerable over-estimation of clouds and ice (Kraatz et al., 2017). This recent study also reported that there are substantial differences between the MODIS and VIIRS cloud-mask products, especially in the presence of ice. In addition, owing to the fact that the VIIRS and MODIS acquisitions happen at different times of day, the clouds may not be in the same place during the MODIS and VIIRS overpasses, and thus can not be directly compared. 


\begin{tabular}{|c|c|c|c|c|}
\hline \multirow{2}{*}{ Lake } & \multicolumn{2}{|c|}{ VIIRS } & \multicolumn{2}{c|}{ MODIS } \\
\cline { 2 - 5 } & Clean & Mixed & Clean & Mixed \\
\hline Sihl & 45 & 63 & 115 & 104 \\
Sils & 11 & 37 & 33 & 64 \\
Silvaplana & 9 & 24 & 21 & 42 \\
St. Moritz & 0 & 11 & 4 & 19 \\
\hline
\end{tabular}

Table 6: The number of clean and mixed pixels per acquisition per lake, for VIIRS (375m GSD) and MODIS (250m GSD).

For VIIRS, four levels of cloud confidence are provided: confidently cloudy, probably cloudy, probably clear and confidently clear. Lake ice phenology using VIIRS is only retrieved for pixels that are confidently clear. For MODIS, a binary cloud-mask is generated from the 48-bit cloud-mask product ( $M O D 35$ ) following a conservative approach, combining the cloudy and uncertain clear bits. We have observed errors in the cloud-masks of both sensors, which are one of the main error sources in the final classification results. However, we still use them as they are. Cloud masking is outside the scope of the present project. Our results thus provide a realistic estimate of what can be achieved with the existing cloud-masks, and a lower bound for a processing chain with improved cloud-masks.

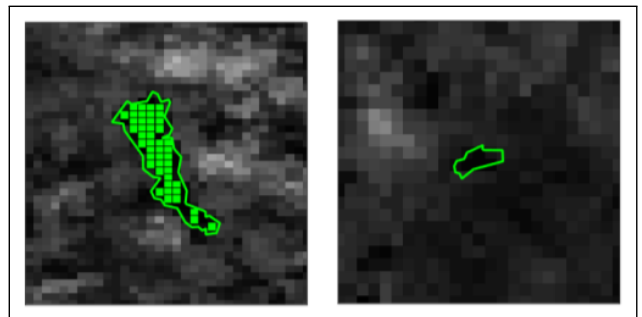

Figure 3: Outlines of the two target lakes (left: Sihl, right: St. Moritz) overlaid on VIIRS data. The clean pixels are shown as green squares. There exist no clean pixel for St. Moritz.

Cloud-free Clean Pixels. As shown in Figure 3, only the clean pixels which lie completely inside the lakes are analyzed. To determine the clean pixels, the lake outlines are corrected for absolute geolocation errors using the values on Table 5, and backprojected on to VIIRS channel $I_{2}(375 \mathrm{~m})$, respectively MODIS channel $B_{2}(250 \mathrm{~m})$. The details of the pixel separation for all four lakes are shown on Table 6. Since MODIS has channels with better spatial resolution compared to VIIRS, it is relatively more useful in the analysis of smaller lakes like St. Moritz, which is clearly an advantage over VIIRS.

\subsection{Lake Ice Detection}

The flow chart of our lake ice detector is shown in Figure 4. The various image channels are extracted from the raw satellite data after re-sampling and re-projection to the UTM32N coordinate system. The generalized lake outlines are back-projected on to the image space and corrected for absolute geolocation error. The corrected outlines are then used to identify the clean pixels. Using the binary cloud-mask generated from the cloud-mask product, the cloudy (clean) pixels are also discarded. These steps are identical for both sensors, for MODIS we additionally up-sample the low-resolution bands (500m and $1000 \mathrm{~m} \mathrm{GSD}$ ) to $250 \mathrm{~m}$ resolution, with bilinear interpolation. Lake ice detection is cast as a two class (frozen, non-frozen) semantic segmentation problem. To select the channels with best inter-class separability, the cloudfree, clean pixels are fed into xgboost (Chen and Guestrin, 2016). While that method in principle already performs classification, we only use the built-in variable selection. The selected channels are then passed to a SVM (Cortes and Vapnik, 1995) for classification (respectively, regression), since it delivered better results

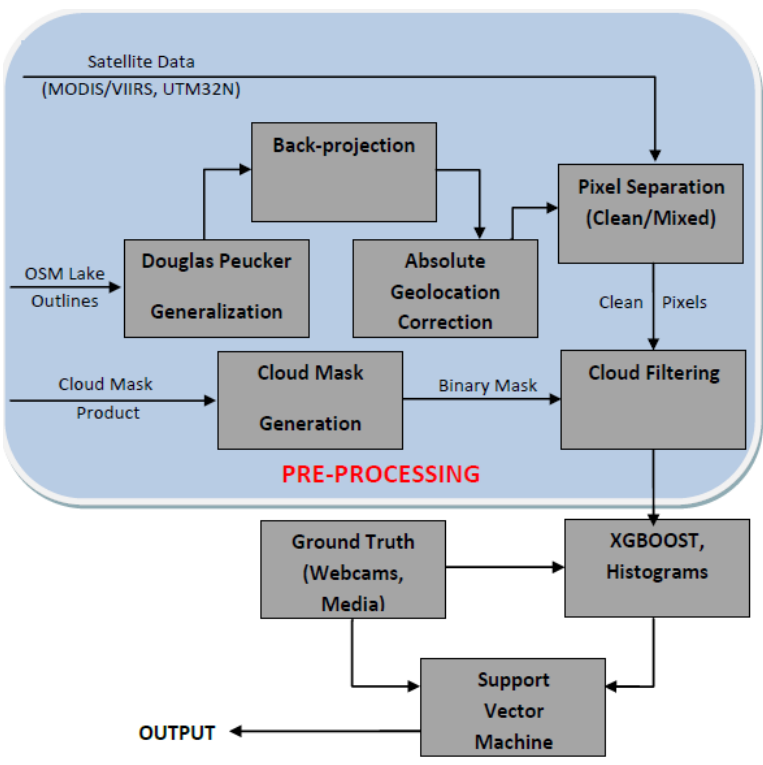

Figure 4: Flowchart of the lake ice detection methodology.

than xgboost itself. For the SVM, the feature vector dimensions are centered and scaled to unit standard deviation. Empirically, a linear kernel is often sufficient, but in some situations it struggles to separate the classes and the Radial Basis Function (RBF) kernel is preferable.

To assess the influence of different channels, we perform a supervised variable importance analysis. The VIIRS channels in Table 2 are analyzed with $x g b o o s t$, and the same analysis is done for the MODIS bands in Table 1. xgboost is a boosting method with shallow decision trees as weak learners. It internally computes variable importance, conditioned on the training labels. The F-score denotes the relative contribution of each channel to the model, calculated from the channel's contribution to individual trees. A higher value implies that the channel is more important to make correct predictions. The results of the analysis for VIIRS are shown in Figure 6. It can be inferred that, for the data tested in winter 2016-17, the channel $I_{1}$ has the best inter-class separability among the VIIRS imagery channels. As a second check, we also use the histograms of the target classes in all the channels to verify the channel selection. For lake Sihl, the histograms of both frozen and non-frozen data in all VIIRS channels are shown in Figure 5. It can be seen that both $I_{1}$ and $I_{2}$ have high inter-class separability. Since the two channels are spectrally close, see Table 4 , and highly correlated, automatic variable selection chooses only one of them. A similar analysis for MODIS was already performed in Tom et al. (2017).

\section{EXPERIMENTS, RESULTS AND DISCUSSION}

Unless mentioned differently, all results reported in the paper are averages from 4 -fold cross-validation. Each lake is trained and tested individually.

\subsection{VIIRS, Winter 2016-17}

Quantitative Results. Different combinations of channels were tested as feature vector and the best results are reported on Table 7. It can be seen that, even with a linear kernel, SVM delivered near perfect results for the period winter 2016-17. The best result was obtained when all five image channels were used, but, as predicted by xgboost, even the reflective channel $I_{1}$ alone is enough to distinguish frozen pixels from non-frozen ones almost perfectly. We also tried the combinations of all reflective bands $\left(I_{1}, I_{2}, I_{3}\right)$ and thermal bands $\left(I_{4}, I_{5}\right)$. The reflective channels 


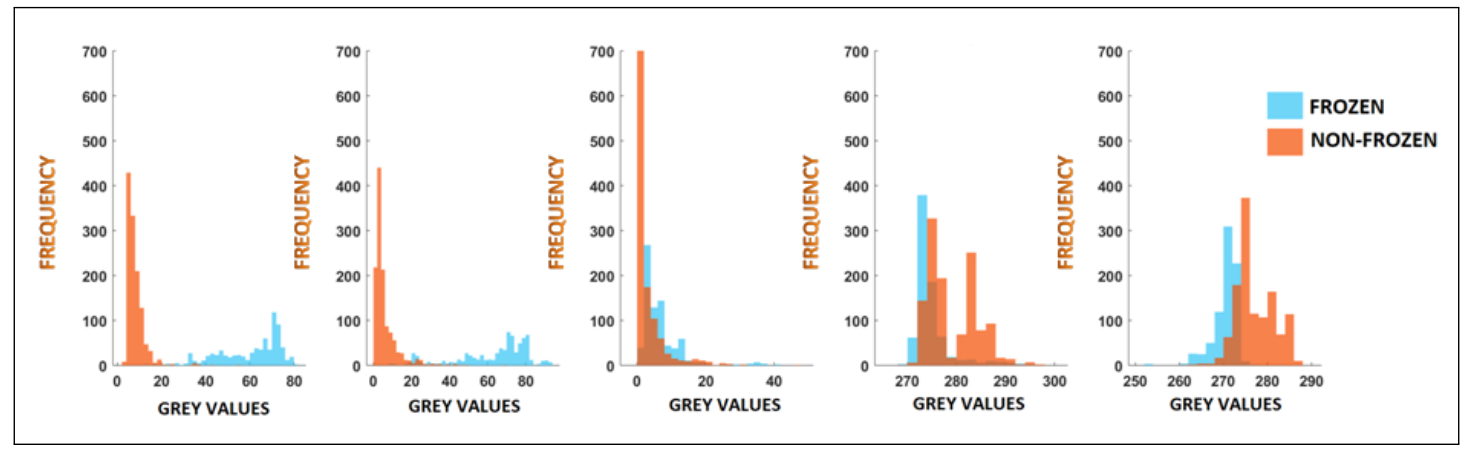

Figure 5: Grey-value histograms of all VIIRS channels (from left to right : $I_{1}, I_{2}, I_{3}, I_{4}, I_{5}$ ) for lake Sihl. Non-frozen data is from images in October 2016 and first half of December 2016. Frozen data refers to images from February 2017. Best viewed on screen.

\begin{tabular}{|c|l|l|c|c|c|c|c|c|c|c|}
\hline \multirow{2}{*}{ Feature Vector } & \multirow{2}{*}{ Sensor } & \multirow{2}{*}{ Kernel } & \multicolumn{2}{|c|}{ Sihl } & \multicolumn{2}{c|}{ Sils } & \multicolumn{2}{c|}{ Silvaplana } & \multicolumn{2}{c|}{ St. Moritz } \\
\cline { 4 - 10 } & & & accuracy & kappa & accuracy & kappa & accuracy & kappa & accuracy & kappa \\
\hline$I_{1}$ & VIIRS & Linear & $98.8 \%$ & 0.974 & $99.8 \%$ & 0.995 & $98.8 \%$ & 0.973 & - & - \\
$I_{4}, I_{5}$ & VIIRS & Linear & $91.2 \%$ & 0.821 & $95.2 \%$ & 0.893 & $96.7 \%$ & 0.925 & - & - \\
$I_{1}, I_{2}, I_{3}$ & VIIRS & Linear & $\mathbf{9 9 . 3 \%}$ & $\mathbf{0 . 9 8 5}$ & $\mathbf{1 0 0 . 0 \%}$ & $\mathbf{1 . 0}$ & $99.1 \%$ & 0.978 & - & - \\
$I_{1}, I_{2}, I_{3}, I_{4}, I_{5}$ & VIIRS & Linear & $\mathbf{9 9 . 3 \%}$ & $\mathbf{0 . 9 8 5}$ & $\mathbf{1 0 0 . 0 \%}$ & $\mathbf{1 . 0}$ & $\mathbf{9 9 . 5 \%}$ & $\mathbf{0 . 9 8 9}$ & - & - \\
\hline$B_{2}$ & MODIS & Linear & $97.5 \%$ & 0.941 & $99.3 \%$ & 0.985 & $98.8 \%$ & 0.975 & $\mathbf{1 0 0 . 0 \%}$ & $\mathbf{1 . 0}$ \\
$B_{22}$ & MODIS & Linear & $89.9 \%$ & 0.751 & $81.1 \%$ & 0.600 & $75.0 \%$ & 0.490 & $72.7 \%$ & 0.474 \\
$B_{2}, B_{22}$ & MODIS & Linear & $99.2 \%$ & 0.981 & $\mathbf{1 0 0 . 0 \%}$ & $\mathbf{1 . 0}$ & $\mathbf{1 0 0 . 0 \%}$ & $\mathbf{1 . 0}$ & $\mathbf{1 0 0 . 0 \%}$ & $\mathbf{1 . 0}$ \\
all 12 bands & MODIS & Linear & $\mathbf{9 9 . 9 \%}$ & $\mathbf{0 . 9 9 5}$ & $\mathbf{1 0 0 . 0 \%}$ & $\mathbf{1 . 0}$ & $\mathbf{1 0 0 . 0 \%}$ & $\mathbf{1 . 0}$ & $\mathbf{1 0 0 . 0 \%}$ & $\mathbf{1 . 0}$ \\
\hline
\end{tabular}

Table 7: Results from 2016-17 for both VIIRS and MODIS data: Comparison of 4-fold cross-validated SVM results with different channel combinations as feature vector. The overall accuracy and kappa coefficients are both given.

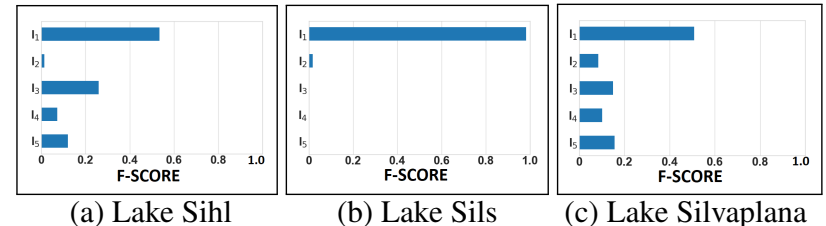

Figure 6: Relative F-scores for the VIIRS channels, as computed with xgboost. Best viewed on screen.

performed markedly better than the thermal ones. It can bee seen in Figure 5 that the separation between snow and water is where the reflective channels $I_{1}$ and $I_{2}$ outperform the thermal channels $I_{4}$ and $I_{5}$.

Qualitative Results. Selected qualitative results for VIIRS data are shown in Figure 7. The first and second rows display results of SVM classification and regression, respectively. In the second row, red colour means higher likelihood of non-frozen, blue colour means higher likelihood of frozen. Results are only shown for cloud-free pixels. For the lake Sils, all the dates were successfully classified. For lakes Sihl and Silvaplana failure cases are also displayed. It can be seen that, for lake Sihl on a nonfrozen day 11.10.2016, some pixels were classified as frozen. These mistakes lie near cloudy pixels, we thus assert that the failures are due to the influence of nearby clouds. The fourth and fifth columns show results for lake Silvaplana on the same frozen date 11.02.2017 at two different acquisition times. In one of the acquisitions (fourth column), a pixel is wrongly classified as nonfrozen. The outlines are overlaid on the reflective channel $I_{1}$ and it can be observed that the pixel appears relatively darker when misclassified. Again there are clouds nearby, seemingly the lower intensity is due to a cloud shadow. A timeline of the pixel-wise results of SVM regression for lake Sihl, as shown in Figure 8, gives additional insights. The acquisitions are plotted in chronological order from left to right on the $\mathrm{x}$-axis. It is notable how noisy the regression results are during the frozen period, compared to the non-frozen counterpart. This can be explained by the fact that the frozen state has a greater radiometric variability (with different states of snow and ice) compared to liquid water.

\subsection{MODIS}

Winter 2016-17. Due to space limitations, only the quantitative results are shown. Different channel combinations, inspired from Tom et al. (2017) have been tested. Like VIIRS, simple linear SVM gave near-perfect results. Hence, experiments with RBF kernel were not conducted. The best results are obtained when all the 12 selected channels are fed into the feature vector. It can be seen that, the reflective channel $B_{2}$ itself is enough to obtain a very good classification result. However, as opposed to the results in Tom et al. (2017), the performance of emissive channel $B_{22}$ alone is not good. Note that here we process additional data from the slightly more challenging December dates. For the emissive band $B_{22}$, the grey value statistics of the clean cloud-free pixels from winter 2016-17 are shown in Figure 9. Left and right columns show the statistics including and excluding December 2016 pixels. One can see that the latter show relatively cold temperatures, which intuitively are more easily confused with cold snow/ice pixels from February 2017 in the emissive channels. We note that for the period winter 2016-17, the performance of VIIRS emissive channels $\left(I_{4}, I_{5}\right)$ is also not as good as for reflective channels.

Winter 2011-12. Quantitative results are displayed in Table 8. It can be seen that in this case the RBF kernel works better compared to the linear kernel. For lake Sihl, it is snow vs. water classification. Hence, even the reflective channel $B_{2}$ achieved very good results. Along with snow-covered days, the lakes Sils, Silvaplana and St. Moritz had icy but snow-free days too. This is the reason why the reflective channel $B_{2}$ alone did not perform well (for both RBF and linear kernels) for these three lakes. The performance is still relatively good for lake St. Moritz, which had few days with ice, but no snow on it. In general, adding the emissive band $B_{22}$ solved the problem, and using all bands brought no further improvement. In some cases the performance decreased slightly, likely due to overfitting to our relatively small training set. For lake Sihl, adding $B_{22}$ causes a significant performance 


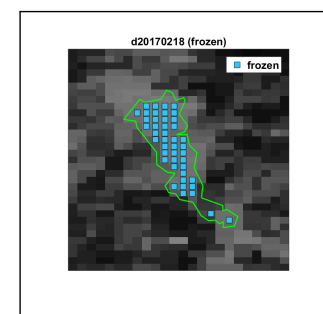

(a) 18.02 .2017

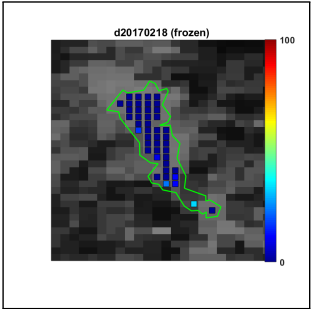

(f) 18.02 .2017

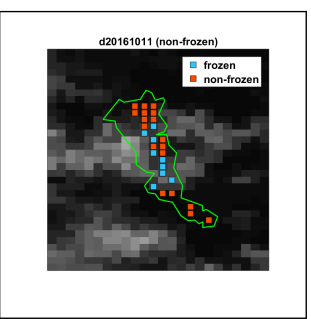

(b) 11.10 .2016

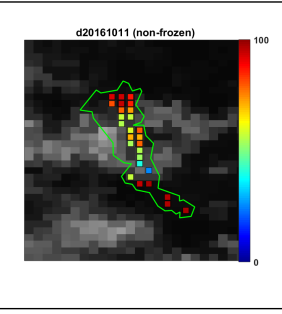

(g) 11.10.2016

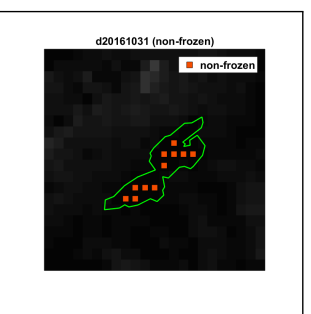

(c) 31.10 .2016

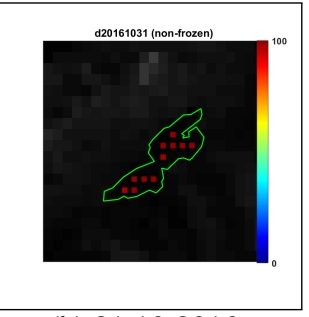

(h) 31.10 .2016

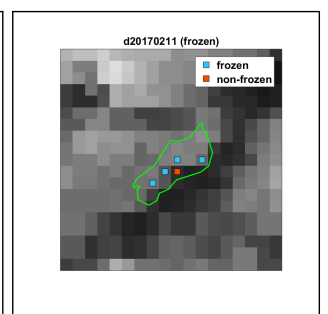

(d) 11.02 .2017

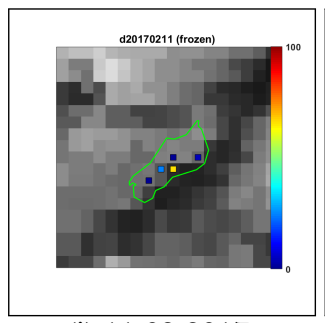

(i) 11.02 .2017

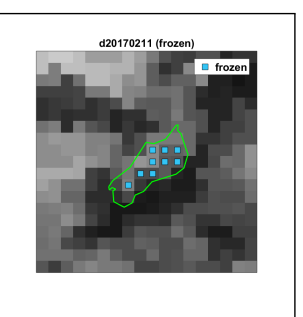

(e) 11.02 .2017

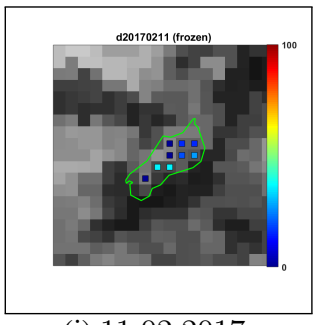

(j) 11.02 .2017

Figure 7: Results from winter 2016-17 on VIIRS data. First and second rows show classification and regression results, respectively. In regression, red means non-frozen and blue frozen. results for cloudy clean pixels are not displayed. Best viewed on screen.

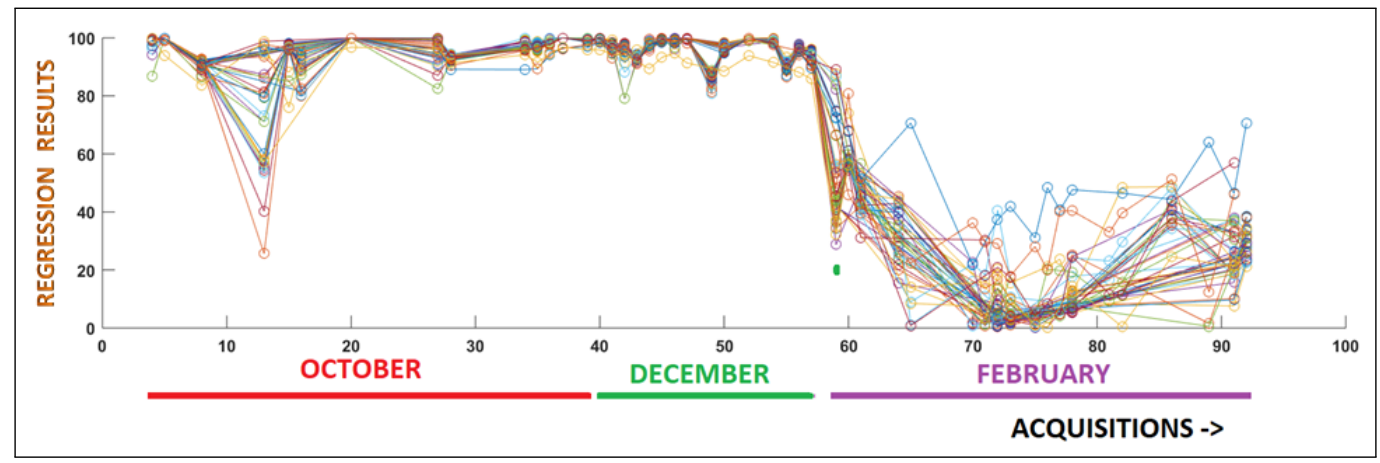

Figure 8: Timeline showing the evolution of the regression values (0: frozen, 100: non-frozen) for each clean pixel (total 45) of lake Sihl on VIIRS data. Each line and circle denote a clean pixel and acquisition respectively. If a pixel is cloudy on an acquisition, the corresponding circle is skipped on the line. The acquisitions on $\mathrm{x}$-axis are shown in the same chronological order as in Figure 2 . The outliers at about acquisition 13 are due to the problem shown in Figure $7 \mathrm{~b}$ and g. The data gap from 16 December 2016 till 31 January 2017 is skipped. Best viewed on screen.

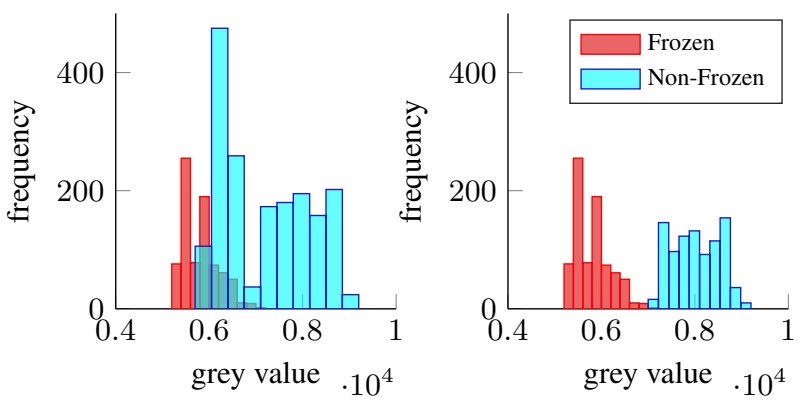

Figure 9: Statistics of the clean cloud-free pixels in MODIS emissive channel $B_{22}$ for lake Sihl from winter 2016-17. Left and right columns show the statistics with pixels from December 2016 included and excluded respectively.

drop, because of two outlier dates (frozen date 24.02.2012 and non-frozen date 20.03.2012). These two dates have similar grey value distribution in the emissive band, such that the data is no longer linearly separable. The RBF kernel alleviates this problem, but does not solve it completely.

\section{Miscellaneous Experiments.}

MODIS data from both winters (2011-12 and 2016-17) were combined into one dataset and processed. The results are shown on Table 9. Overall, the combined dataset clearly improves the correctness of the result. Since both the training and test sets are a mix of images from both winters, we have effectively increased the amount of training data, while the difficulty of the problem remains about the same. Hence, the experiment provides an indication that the previous datasets were still not large enough for the performance to saturate, and the classification can be expected to become more reliable if it receives more training data.

As a second experiment, for each lake, an SVM model is trained using all the images from winter 2011-12, and then tested on all images from 2016-17. This experiment assesses the stability of the classifier over time, respectively how well a classifier trained on one winter transfers to another one. The results are displayed on Table 10. They should be compared with Table 7. While there is a noticeable drop in accuracy $(\approx 5 \%$ for lake Sihl, Sils and Silvaplana, twice as much for St. Moritz) the ice classification is still correct for $90 \%$ or more of the pixels, on all lakes.

In the final experiment, we train the SVM model on the data from three lakes (both winters 2011-12 and 2016-17) and test on the fourth lake. The results are displayed on Table 11. This experiment serves to assess the transferability of the trained model across lakes. It can be seen that our model fairs well in generalizing from one lake to another. It can thus be expected that data for a limited set of lakes, from few winters, will be enough to train a classifier which can then be applied to any unseen image. This would clearly be a valuable asset for an operational system. A deeper investigation requires additional data and is thus left for future work. 
ISPRS Annals of the Photogrammetry, Remote Sensing and Spatial Information Sciences, Volume IV-2, 2018 ISPRS TC II Mid-term Symposium “Towards Photogrammetry 2020”, 4-7 June 2018, Riva del Garda, Italy

\begin{tabular}{|c|c|c|c|c|c|c|c|c|c|}
\hline \multirow{2}{*}{ Feature Vector } & \multirow{2}{*}{ SVM Kernel } & \multicolumn{2}{|c|}{ Sihl } & \multicolumn{2}{c|}{ Sils } & \multicolumn{2}{c|}{ Silvaplana } & \multicolumn{2}{c|}{ St. Moritz } \\
\cline { 3 - 9 } & & accuracy & kappa & accuracy & kappa & accuracy & kappa & accuracy & kappa \\
\hline$B_{2}$ & Linear & $99.2 \%$ & 0.983 & $81.1 \%$ & 0.642 & $79.1 \%$ & 0.592 & $96.0 \%$ & 0.917 \\
$B_{22}$ & Linear & $82.4 \%$ & 0.653 & $97.7 \%$ & 0.953 & $93.2 \%$ & 0.843 & $94.6 \%$ & 0.898 \\
$B_{2}, B_{22}$ & Linear & $91.5 \%$ & 0.831 & $\mathbf{9 8 . 5 \%}$ & $\mathbf{0 . 9 6 9}$ & $99.9 \%$ & 0.997 & $\mathbf{1 0 0 . 0 \%}$ & $\mathbf{1 . 0}$ \\
all 12 bands & Linear & $83.1 \%$ & 0.670 & $98.5 \%$ & 0.969 & $\mathbf{1 0 0 . 0 \%}$ & $\mathbf{1 . 0}$ & $\mathbf{1 0 0 . 0 \%}$ & $\mathbf{1 . 0}$ \\
\hline$B_{2}$ & RBF & $\mathbf{9 9 . 6 \%}$ & $\mathbf{0 . 9 9 2}$ & $81.3 \%$ & 0.632 & $80.1 \%$ & 0.629 & $96.0 \%$ & 0.918 \\
$B_{22}$ & RBF & $98.6 \%$ & 0.973 & $98.1 \%$ & 0.961 & $96.0 \%$ & 0.915 & $92.6 \%$ & 0.844 \\
$B_{2}, B_{22}$ & RBF & $95.1 \%$ & 0.894 & $97.9 \%$ & 0.956 & $99.9 \%$ & 0.998 & $\mathbf{1 0 0 . 0 \%}$ & $\mathbf{1 . 0}$ \\
all 12 bands & RBF & $87.5 \%$ & 0.752 & $\mathbf{9 8 . 5 \%}$ & $\mathbf{0 . 9 7 0}$ & $99.6 \%$ & 0.992 & $\mathbf{1 0 0 . 0 \%}$ & $\mathbf{1 . 0}$ \\
\hline
\end{tabular}

Table 8: Results from winter 2011-12 for MODIS data. Comparison of 4-fold cross-validated SVM results. The ground truth data displayed on Table 3 is used.

\begin{tabular}{|c|c|c|c|c|c|c|c|c|c|}
\hline \multirow{2}{*}{ Feature Vector } & \multirow{2}{*}{ SVM Kernel } & \multicolumn{2}{|c|}{ Sihl } & \multicolumn{2}{c|}{ Sils } & \multicolumn{2}{c|}{ Silvaplana } & \multicolumn{2}{c|}{ St. Moritz } \\
\cline { 3 - 9 } & & accuracy & kappa & accuracy & kappa & accuracy & kappa & accuracy & kappa \\
\hline$B_{2}$ & Linear & $\mathbf{9 8 . 2 \%}$ & $\mathbf{0 . 9 6 1}$ & $86.1 \%$ & 0.723 & $86.3 \%$ & 0.728 & $97.5 \%$ & 0.949 \\
$B_{22}$ & Linear & $87.7 \%$ & 0.740 & $92.0 \%$ & 0.841 & $86.1 \%$ & 0.721 & $87.7 \%$ & 0.751 \\
$B_{2}, B_{22}$ & Linear & $95.1 \%$ & 0.90 & $96.5 \%$ & 0.929 & $97.9 \%$ & 0.957 & $97.9 \%$ & 0.958 \\
all 12 bands & Linear & $94.9 \%$ & 0.90 & $\mathbf{9 9 . 8 \%}$ & $\mathbf{0 . 9 9 6}$ & $99.7 \%$ & 0.994 & $\mathbf{1 0 0 . 0 \%}$ & $\mathbf{1 . 0}$ \\
\hline$B_{2}$ & RBF & $97.7 \%$ & 0.951 & $86.6 \%$ & 0.733 & $85.9 \%$ & 0.725 & $97.6 \%$ & 0.949 \\
$B_{22}$ & RBF & $87.5 \%$ & 0.708 & $91.7 \%$ & 0.835 & $86.7 \%$ & 0.734 & $89.8 \%$ & 0.791 \\
$B_{2}, B_{22}$ & RBF & $96.4 \%$ & 0.927 & $97.3 \%$ & 0.946 & $97.0 \%$ & 0.939 & $97.9 \%$ & 0.958 \\
all 12 bands & RBF & $92.2 \%$ & 0.831 & $99.0 \%$ & 0.979 & $\mathbf{9 9 . 9 \%}$ & $\mathbf{0 . 9 9 9}$ & $94.5 \%$ & 0.887 \\
\hline
\end{tabular}

Table 9: MODIS results on the combined data from both winter 2011-12 and winter 2016-17.

\section{CONCLUSION AND OUTLOOK}

We proposed a methodology for lake ice detection from lowresolution optical/thermal satellite data. While we have concentrated on lakes in Switzerland, the methodology is generic and should be applicable elsewhere too. We have shown that a simple, transparent classification approach achieves high accuracy with both MODIS and VIIRS sensors, for all tested lakes. On one hand, compared to MODIS, VIIRS has better temporal resolution, and therefore also (on average) shorter data gaps due to cloudy images. On the other hand, the better spatial resolution of MODIS (250m) makes it possible to analyze smaller lakes such as St. Moritz. We have demonstrated that our approach gives consistent results over multiple winters, and that it generalizes fairly well from one winter to another. We have also shown that the model generalizes well across lakes.

A limiting factor is the coarse resolution of the images, which makes it difficult to process small lakes. Moreover, it restricts the size of the dataset on which we report our results. Another issue is the uncertainty of the ground truth. Webcams typically do not cover the entire water surface, and it is often difficult to manually interpret the state of the lake. Collection of large-scale in-situ ground truth would obviously solve this problem, but is time-consuming and expensive. We plan to enrich the current ground truth with a combination of in-situ temperature measurements and interpretation of high-resolution satellite images such as Sentinel 2, Landsat etc. Finally, the standard cloud-masks of both MODIS and VIIRS are not perfect and a significant source of error at the observed, high accuracies of ice detection. In particular, some actual cloud pixels are not detected, which are then classified as snow or ice.

What is still missing is the processing of the relatively short freezing and thawing periods, where frozen and non-frozen pixels co-exist on the same lake. We are planning to process such challenging dates, as well as additional winters and more lakes, in future work. Especially on small lakes, the classification will also have to deal with mixed pixels to correctly recover the proportion of ice. In the same vein, processing not only the "clean" pixels, but also those partially inside the lake boundary may give further information near the lake border, where freezing typically starts. We also expect that multi-temporal analysis will further improve the results, since freezing and thawing cycles are relatively smooth over periods of several days.

\section{ACKNOWLEDGEMENTS}

This work is part of the project Integrated Monitoring of Ice in Selected Swiss Lakes funded by Swiss Federal Office of Meteorology and Climatology MeteoSwiss in the framework of GCOS Switzerland.

\section{References}

Bernhardt, J., Engelhardt, C., Kirillin, G. and Matschullat, J., 2012. Lake ice phenology in Berlin-Brandenburg from 19472007: observations and model hindcasts. Climatic Change 112(3), pp. 791-817.

Brown, L. C. and Duguay, C. R., 2012. Modelling Lake Ice Phenology with an Examination of Satellite-Detected Subgrid Cell Variability. Advances in Meteorology 6(2), pp. 431-446.

Chen, T. and Guestrin, C., 2016. XGBoost: A Scalable Tree Boosting System. In: Proc. 22nd ACM SIGKDD International Conference on Knowledge Discovery and Data Mining, pp. 785-794.

Cooley, S. W. and Pavelsky, T. M., 2016. Spatial and temporal patterns in Arctic river ice breakup revealed by automated ice detection from MODIS imagery. Remote Sensing of Environment 175 , pp. $310-322$.

Cortes, C. and Vapnik, V., 1995. Support-vector networks. Machine Learning 20(3), pp. 273-297.

Dörnhöfer, K. and Oppelt, N., 2016. Remote sensing for lake research and monitoring - Recent advances. Ecological Indicators 64, pp. 105-122. 
ISPRS Annals of the Photogrammetry, Remote Sensing and Spatial Information Sciences, Volume IV-2, 2018 ISPRS TC II Mid-term Symposium “Towards Photogrammetry 2020”, 4-7 June 2018, Riva del Garda, Italy

\begin{tabular}{|c|c|c|c|c|c|c|c|c|c|}
\hline \multirow{2}{*}{ Feature Vector } & \multirow{2}{*}{ SVM Kernel } & \multicolumn{2}{|c|}{ Sihl } & \multicolumn{2}{c|}{ Sils } & \multicolumn{2}{c|}{ Silvaplana } & \multicolumn{3}{c|}{ St. Moritz } \\
\cline { 3 - 10 } & & accuracy & kappa & accuracy & kappa & accuracy & kappa & accuracy & kappa \\
\hline$B_{2}, B_{22}$ & Linear & $\mathbf{9 6 . 3 \%}$ & $\mathbf{0 . 9 0 9}$ & $88.4 \%$ & 0.744 & $90.5 \%$ & 0.808 & $86.4 \%$ & 0.732 \\
all 12 bands & Linear & $92.9 \%$ & 0.821 & $\mathbf{9 7 . 4 \%}$ & $\mathbf{0 . 9 4 4}$ & $\mathbf{9 4 . 1 \%}$ & $\mathbf{0 . 8 7 8}$ & $\mathbf{8 9 . 8 \%}$ & $\mathbf{0 . 7 9 6}$ \\
\hline$B_{2}, B_{22}$ & RBF & $96.0 \%$ & 0.902 & $92.6 \%$ & 0.844 & $90.6 \%$ & 0.812 & $81.8 \%$ & 0.648 \\
all 12 bands & RBF & $87.6 \%$ & 0.672 & $86.1 \%$ & 0.722 & $81.3 \%$ & 0.638 & $62.5 \%$ & 0.320 \\
\hline
\end{tabular}

Table 10: Generalization across winters: MODIS results when the SVM is trained with data from winter 2011-12 and tested with data from winter 2016-17.

\begin{tabular}{|c|c|c|c|c|c|c|c|c|c|}
\hline \multirow{2}{*}{ Feature Vector } & \multirow{2}{*}{ SVM Kernel } & \multicolumn{2}{|c|}{ Sihl } & \multicolumn{2}{c|}{ Sils } & \multicolumn{2}{c|}{ Silvaplana } & \multicolumn{3}{c|}{ St. Moritz } \\
\cline { 3 - 9 } & & accuracy & kappa & accuracy & kappa & accuracy & kappa & accuracy & kappa \\
\hline$B_{2}, B_{22}$ & Linear & $\mathbf{9 8 . 1 \%}$ & $\mathbf{0 . 9 6}$ & $97.4 \%$ & 0.949 & $97.9 \%$ & 0.959 & $\mathbf{9 7 . 0 \%}$ & $\mathbf{0 . 9 4}$ \\
all 12 bands & Linear & $96.6 \%$ & 0.93 & $\mathbf{9 8 . 7 \%}$ & $\mathbf{0 . 9 7 4}$ & $\mathbf{9 8 . 2 \%}$ & $\mathbf{0 . 9 6 3}$ & $96.6 \%$ & 0.931 \\
\hline
\end{tabular}

Table 11: Generalization across lakes: MODIS results when the SVM is trained on three lakes and tested on the fourth combining data from both winter 2011-12 and winter 2016-17.

Kocaman Aksakal, S., 2013. Geometric Accuracy Investigations of SEVIRI High Resolution Visible (HRV) Level 1.5 Imagery. Remote Sensing 5(5), pp. 2475-2491.

Korzeniowska, K. and Korup, O., 2017. Object-Based Detection of Lakes Prone to Seasonal Ice Cover on the Tibetan Plateau. Remote Sensing 9(4), pp. 339.

Kraatz, S., Khanbilvardi, R. and Romanov, P., 2017. A Comparison of MODIS/VIIRS Cloud Masks over Ice-Bearing River: On Achieving Consistent Cloud Masking and Improved River Ice Mapping. Remote Sensing 9(3), pp. 229.

Liu, Y., Key, J. and Mahoney, R., 2016. Sea and Freshwater Ice Concentration from VIIRS on Suomi NPP and the Future JPSS Satellites. Remote Sensing 8(6), pp. 523.

Maslanik, J. A. and Barry, R. G., 1987. Lake ice formation and breakup as an indicator of climate change: Potential for monitoring using remote sensing techniques. Proceedings of the IAHS Symposium, Vancouver, Canada 168, pp. 153-161.

Nishihama, M., Wolfe, R., Solomon, D., Patt, F., Blanchette, J., Fleig, A. and Masuoka, E., 1997. MODIS Level 1A Earth Location: Algorithm Theoretical Basis Document Version 3.0. Technical report, NASA Goddard Space Flight Center.

Palecki, M. A. and Barry, R. G., 1986. Freeze-up and break-up of lakes as an index of temperature changes during the transition seasons: a case study for Finland. Journal of Climate and Applied Meteorology 25(7), pp. 893-902.

Rösel, A., Kaleschke, L. and Birnbaum, G., 2012. Melt ponds on Arctic sea ice determined from MODIS satellite data using an artificial neural network. Cryosphere 6(2), pp. 431-446.

Sánchez-López, G., Hernández, A., Pla-Rabes, S. and Toro, M., 2015. The effects of the NAO on the ice phenology of Spanish alpine lakes. Climatic Change 130(2), pp. 101-113.

Soja, A. M., Kutics, K., Maracek, K., Molnár, G. and Soja, G., 2014. Changes in ice phenology characteristics of two central European steppe lakes from 1926 to 2012 - Influences of local weather and large scale oscillation patterns. Climatic Change 126(1-2), pp. 119-133.

Spencer, P., Miller, A. E., Reed, B. and Budde, M., 2008. Monitoring lake ice seasons in southwest Alaska with MODIS images. In: Pecora 17 Conference: The Future of Land Imaging Going Operational.
Suomi National Polar-orbiting Partnership Mission, n.d. https://eoportal.org/web/eoportal/ satellite-missions/s/suomi-npp. Retrieved January $11,2018$.

Sütterlin, M., Duguay-Tetzlaff, A. and Wunderle, S., 2017. Toward a Lake Ice Phenology Derived from VIIRS Data. In: EGU General Assembly.

Terra Mission (EOS/AM-1), n.d. https://eoportal.org/ web/eoportal/satellite-missions/t/terra. Retrieved January 11, 2018.

Tom, M., Lanaras, C., Baltsavias, E. and Schindler, K., 2017. Ice detection in swiss lakes using modis data. In: Proceedings of the 38th Asian Conference on Remote Sensing.

Trishchenko, A. P. and Ungureanu, C., 2017. Intercomparison of MODIS and VIIRS Results for Mapping Summer Minimum of Snow and Ice (MSI) Extent Over Canadian Landmass. In: 8th EARSeL workshop on Land Ice and Snow.

Trishchenko, A. P., Kostylev, V. and Whalen, D., 2017. Probabilistic Approach for Mapping Landfast and Sea Ice Extent in the Canadian Arctic Archipelago from MODIS at 250m Spatial Resolution. In: 8th EARSeL workshop on Land Ice and Snow.

Trishchenko, A. P., Leblanc, S. G., Wang, S., Li, J., Ungureanu, C., Luo, Y., Khlopenkov, K. V. and Fontana, F., 2016. Variations of Annual Minimum Snow and Ice Extent over Canada and Neighboring Landmass Derived from MODIS 250-m Imagery for 2000-2014. Canadian Journal of Remote Sensing 42(3), pp. 214-242.

Tschudi, M. A., Maslanik, J. A. and Perovich, D. K., 2008. Derivation of melt pond coverage on Arctic sea ice using MODIS observations. Remote Sensing of Environment 112(5), pp. 2605-2614

VIIRS with Pytroll, n.d. http://pytroll.readthedocs.io/ en/latest/quickstart_viirs.html. Retrieved January 11,2018

Weber, H., Riffler, M., Nõges, T. and Wunderle, S., 2016. Lake ice phenology from AVHRR data for European lakes: An automated two-step extraction method. Remote Sensing of Environment 174 , pp. 329-340.

Wynne, R. H. and Lillesand, T. M., 1993. Satellite observation of lake ice as a climate indicator: Initial results from statewide monitoring in Wisconsin. Photogrammetric Engineering and Remote Sensing 59(6), pp. 1023-1031. 